\title{
Venous thromboembolism in infancy and childhood
}

\author{
D. R. B. JONES and I. M. C. MACINTYRE \\ From the Department of Clinical Surgery, Western General Hospital, Edinburgh
}

\begin{abstract}
Jones, D. R. B., and Macintyre, I. M. C. (1975). Archives of Disease in Childhood, 50, 153. Venous thromboembolism in infancy and childhood. A retrospective study of Scottish hospital inpatients aged 15 years or less has revealed, over a 4-year period, 36 cases of venous thrombosis or phlebitis. Of these 10 were considered to have deep venous thrombosis; 2 cases were associated with obvious pulmonary embolism and 2 cases developed chronic venous insufficiency. Changes in the coding allocations are proposed. There are 'high risk' situations in childhood disease in which a diagnosis of venous thromboembolic disease should be considered and investigated.
\end{abstract}

Deep vein thrombosis and its sequelae are common in adults and the incidence is increasing (Morrell and Dunnill, 1968; Vessey, 1973). It is generally believed that the disorder is rare in childhood though published reports are few. Having recently seen an 8-year-old child with iliofemoral thrombosis occurring after injury we were prompted to review venous thromboembolic disease in Scottish hospital inpatients under 15 years of age.

\section{Methods}

All inpatients in Scottish hospitals have up to three diagnoses coded on discharge. The diagnostic codes relevant to venous thromboembolism in the eighth (1968) revision of the International Classification of Disease are, 450 pulmonary embolism, 451 phlebitis and thrombophlebitis, 453 other venous embolism and thrombosis.

The Scottish Home and Health Department supplied a list of all patients discharged from 1968 to 1971 inclusive who had one or more of these codes entered, which identified each patient by hospital, unit number, and age only. All patients aged 15 years or under were selected from the list. In each case the consultant concerned was approached and permission sought to examine the case notes.

\section{Results}

Information was requested in 49 cases and was made available in 48 . However, 12 further cases were omitted because of errors in clinical and computer data. Analysis of the diagnoses in the remaining 36 cases is shown in the Table.

Received 2 July 1974.
TABLE

Clinical diagnoses

\begin{tabular}{l|r}
\hline Renal vein thrombosis & 12 \\
Caval thrombosis following ventriculo-atrial shunt & 4 \\
Cerebral thrombophlebitis & 3 \\
Umbilical vein thrombosis & 2 \\
Pulmonary thrombosis in infancy & 2 \\
Axillary vein thrombosis & 2 \\
Jugular vein thrombosis & 1 \\
Deep venous thrombosis pulmonary embolism & 10 \\
\hline Total & 36 \\
\hline
\end{tabular}

Renal vein thrombosis is well recognized in childhood and appears here because no separate coding is provided in the International Classification of Diseases. The cases of umbilical vein thrombosis and thrombosis of the superior vena cava were all associated with prolonged venous catheterization, the latter following ventriculoatrial shunts. Cerebral thrombophlebitis is specifically excluded from these diagnostic codes, and these cases should have been allotted ICD code 433. The 2 cases of pulmonary arterial thrombosis were associated with severe metabolic disease in neonates. In each case the diagnosis was made only at post mortem, and was regarded as an incidental finding. The diagnoses of axillary vein thrombosis were based on clinical grounds and were not supported by phlebography.

There remain 10 cases in which the clinician had made a diagnosis of pulmonary embolism, superficial or deep vein thrombosis of the lower limb. 
Case 1. Male aged 2 years. After cardiac catheterization via the femoral vein he developed a cold swollen blue leg. No phlebogram. Femoral venous thrombectomy performed. No evidence of pulmonary embolism. Survived.

Case 2. Female aged 2 years. Developed a clinically recognized major pulmonary embolism after a fractured femoral shaft. She died before phlebography or treatment was possible. Necropsy confirmed death due to massive pulmonary embolism.

Case 3. Female aged 3 years. A banding procedure for an anomalous pulmonary artery was followed by fatal pulmonary embolism which was diagnosed clinically. Necropsy confirmed the embolism and showed the source of thrombus to be the left pulmonary artery.

Case 4. Female aged 11 years. Stevens-Johnson syndrome. During prolonged immobilization she showed clinical features of deep vein thrombosis in the leg. No phlebogram performed. No evidence of pulmonary embolism. No anticoagulants given. Survived.

Case 5. Male aged 13 years. This patient developed clinical features of deep vein thrombosis of the leg after appendicectomy. No phlebogram performed. Resolved with anticoagulant therapy. No evidence of pulmonary embolism. Survived.

Case 6. Female aged 14 years. Complained of spontaneous leg pain unrelated to trauma or operation. No phlebogram performed. No evidence of pulmonary embolism. Clinical suspicion of 'venous thrombosis'. No specific treatment. Survived.

Case 7. Female aged 14 years. One month after meniscectomy, complicated by infection, this patient developed clinical features of deep vein thrombosis (DVT). No phlebogram performed. Resolved on anticoagulants. No evidence of pulmonary embolism.

Case 8. Female aged 14 years. 4 days after minor knee trauma she developed clinical features of DVT. No phlebogram performed. Resolved on anticoagulants. No evidence of pulmonary embolism.

Case 9, Male aged 14 years. At the age of 18 months he had had encephalitis with transient hemiplegia. He subsequently presented with chronic swelling in the lower limb with dilatation of superficial veins. Phlebography performed at the age of 12 years showed occlusions from superficial femoral to the common iliac veins. He was treated by sapheno-saphenous cross-over bypass with good result. No evidence of pulmonary embolism.

Case 10. Male aged 15 years. 15 days after appendicectomy he developed clinical features of iliofemoral DVT. No phlebogram or anticoagulants at this time. He subsequently showed the features of chronic venous insufficiency. Phlebography at this time showed thrombus extending from external iliac vein to inferior vena cava. No evidence of pulmonary embolism. Survived.

\section{Discussion}

It is generally believed that the pattern of venous thromboembolism commonly seen in adults is very rare in childhood. As a consequence the diagnosis may be overlooked and specific diagnostic techniques may not be employed.

In adults increased awareness and improved diagnosis have shown that the disorder is more common than was previously supposed. Moreover, the incidence has been shown to be increasing (Vessey, 1973). However, we have been unable to find any reviews of venous thromboembolism in childhood in published reports in English in the past decade. No conclusions can be drawn as to whether the incidence is increasing.

Zuschlag (1947), in a major study of pulmonary infarction in childhood, reviewed 74 cases from published reports. The majority of these were associated with cachexia or infection but only 24 were known to have had thrombi elsewhere, principally in the cerebral sinuses with only 3 thrombi in 'peripheral veins'. He reported a further 38 cases from the Mayo Clinic, the majority associated with infection of which 16 followed operation. In 8 thrombus was found in the iliofemoral segment. Though 'most cases' were considered to be due to embolism, the difficulty in differentiation between pulmonary artery thrombosis and pulmonary embolism was emphasized.

Stevenson and Stevenson (1949) reviewed pulmonary embolism in children under the age of 15 years, listing 29 well documented cases of pulmonary embolism from published reports. In only 3 of these was peripheral venous thrombosis described. Emery (1962) found 25 cases of pulmonary embolism among 2000 necropsies at the Sheffield Children's Hospital. Of these 15 were associated with infection particularly of the upper respiratory tract. The commonest sites of origin of the embolus were nasopharyngeal veins and cerebral veins and sinuses. In 4 cases thrombus was found in the inferior vena cava and renal veins. Cases of emboli arising from ventriculo-atrial shunts and congenital heart lesions were excluded from this study.

Pulmonary emboli in children arising from sources other than the veins of the pelvis and lower limbs are well recognized. Renal vein thrombosis is not uncommon in children (Kaufmann, 1958) and extension to the vena cava has been reported (Bruns, 1960). Thrombosis and embolism resulting from umbilical catheterization (Indar, 1959), indwelling venous catheters particularly when these 
are used for parenteral feeding (Haber and Bennington, 1962), ventriculo-atrial shunts (Gabriele and Clark, 1969), and congenital cardiac lesions are all well recognized.

Thrombus in the deep veins of the limbs and pelvic veins does occur in childhood (Bruns, 1960; Haber and Bennington, 1962; Banks, Raftery, and Oram, 1970) but published reports are few. Failure to consider the condition may result in failure to perform appropriate investigations.

In the present study, using the ICD coding allocation and retrospective analysis of case records, we have again encountered difficulty in drawing valid conclusions about the hospital incidence of DVT and pulmonary embolism (Macintyre and Ruckley, 1974). We believe that because it has been shown to be a very common condition thrombosis of the deep veins of the lower limb (including iliac veins) merits a separate ICD code.

Similarly, to avoid confusion, a separate coding category should be allotted to thrombophlebitis of superficial veins of the lower limb. The code number allocated to 'other venous thrombosis' should be subdivided to specify thrombosis affecting renal vein, umbilical vein, or axillary vein.

Even if the accuracy of the coding were improved the true incidence of venous thromboembolism could not be obtained from a retrospective review of this nature. Nevertheless, we have confirmed that venous thrombosis of the type so familiar in adults does occur in children and furthermore that the grave complications of massive pulmonary embolism and chronic venous insufficiency may ensue.

In this series two phlebograms were performed and these only when an established postphlebitic syndrome was apparent. This may reflect unwillingness of the clinician to expose a child to relatively high amounts of radiation, failure to consider thromboembolism as a possible diagnosis, or lack of appreciation of the importance of phlebography as a guide to treatment.

As in adults it may be possible to define high risk situations. The outstanding ones in childhood are sepsis and trauma. Deep vein thrombosis may mimic acute osteomyelitis and indeed may be associated with the latter (Horvath, Brodeur, and Cherry, 1971). We believe that in 'atypical osteomyelitis' with limb swelling, heat, and tenderness, phlebography should be considered.

Pulmonary embolism in childhood may stimulate acute bronchopneumonia and is most frequently diagnosed as such (Kaufmann, 1958). In any child with an indwelling venous catheter unexpected symptoms of lung infection should at once raise the possibility of embolism. Furthermore, in any child with injury or disease elsewhere who develops 'bronchopneumonia' which fails to respond to antibiotic therapy the diagnosis of thromboembolism should be considered and suitably investigated.

Experience of thromboembolic disease in adults leads us to suspect that the number of cases detected by this survey is a gross underestimate of the true incidence of the disease in children.

We thank Dr. J. D. Donnelly of the Scottish Home and Health Department, Research and Intelligence Unit for access to the Scottish Inpatient Diagnostic Index. We also thank all the Medical Records Officers and Consultants who provided case records, and Mr. C. V. Ruckley for helpful advice and encouragement.

D.R.B.J. is supported by the Under-Secretary of State's Advisory Committee on Medical Research grant number K/MRS/19/192.

\section{REFERENCES}

Banks, D. C., Raftery, E. B., and Oram, S. (1970). Thromboembolic pulmonary hypertension of unusual cause in a teenager. British Medical fournal, 3, 563,

Bruns, W. T. (1960). Ascending thrombosis involving inferior vena cava and renal veins. American fournal of Diseases of Children, 99, 276.

Emery, J. L. (1962). Pulmonary embolism in children. Archives of Disease in Childhood, 37, 591.

Gabriele, O. F., and Clark, D. (1969). Calcified thrombus of the superior vena cava. American fournal of Diseases of Children, 117. 325.

Haber, S. L., and Bennington, J. L. (1962). Pulmonary embolism in an infant. Fournal of Pediatrics, 61, 759.

Horvath, F. L., Brodeur, A. E., and Cherry, J. D. (1971). Deep thrombophlebitis associated with acute osteomyelitis. Fournal of Pediatrics, 79, 815.

Indar, R. (1959). The dangers of indwelling polyethylene cannulae in deep veins. Lancet, 1, 284.

Kaufmann, H. J. (1958). Renal vein thrombosis. American fournal of Diseases of Children, 95, 377.

Macintyre, I. M. C., and Ruckley, C. V. (1974). Pulmonary embolism: a clinical and autopsy study. Scottish Medical fournal, $19,20$.

Morrell, M. T., and Dunnill, M. S. (1968). The post-mortem incidence of pulmonary embolism in a hospital population. British fournal of Surgery, 55, 347.

Stevenson, G. F., and Stevenson, F. L. (1949). Pulmonary embolism in childhood. Fournal of Pediatrics, 34, 62.

Vessey, M. P. (1973). The epidemiology of venous thromboembolism. In Recent Advances in Thrombosis p. 39 Ed. by L. Poller. Churchill Livingstone, Edinburgh and London.

Zuschlag, E. (1947). Infection of the lung in children. American fournal of Diseases of Children, 74, 399.

Correspondence to Mr. D. R. B. Jones, Department of Clinical Surgery, Western General Hospital, Edinburgh EH4 2XU. 